\title{
miR-135b-5p promotes gastric cancer progression by targeting CMTM3
}

\author{
MINGDONG LU ${ }^{1 *}$, YINGPENG HUANG ${ }^{*}$, WEIJIAN SUN ${ }^{2}$, PIHONG LI $^{1}$, LIYI LI $^{1}$ and LEPING LI ${ }^{1}$ \\ ${ }^{1}$ Department of General Surgery, Shandong Provincial Hospital Affiliated to Shandong University, \\ Ji'nan, Shandong; ${ }^{2}$ Department of General Surgery, The Second Affiliated Hospital \\ of Wenzhou Medical Univesity, Wenzhou, Zhejiang, P.R. China
}

Received July 21, 2017; Accepted November 30, 2017

DOI: $10.3892 /$ ijo.2017.4222

\begin{abstract}
CKLF-like MARVEL transmembrane domain containing 3 (CMTM3) is considered to be a tumor suppressor gene in multiple types of malignancies. Previous studies have indicated that CMTM3 suppresses metastasis and epithelialmesenchymal transition (EMT) in gastric cancer. However, its role in gastric cancer cell proliferation has rarely been discussed. Moreover, the regulatory mechanisms of CMTM3 in gastric cancer remain unclear. In this study, RT-qPCR and IHC were used to assess the expression of CMTM3 and miR-135b-5p in gastric cancer tissues and cell lines. We found that the expression of miR-135b-5p was negatively associated with CMTM3 in gastric cancer tissues, and we verified that miR-135b-5p directly targeted CMTM3 in gastric cancer cells by dual-luciferase reporter assay. CCK8 assay, Transwell assay and flow cytometric analysis were conducted to examine the functions of CMTM3 and miR-135b-5p in vitro. Our results demonstrated that the overexpression of CMTM3 or the suppression of miR-135b-5p using an inhibitor suppressed SGC-7901 gastric cancer cell proliferation, invasion and cell cycle progression, and promoted SGC-7901 cell apoptosis. Furthermore, a BALB/c nude mouse subcutaneous xenograft model was used to verify the function of miR-135b-5p and CMTM3. Our results revealed that miR-135b-5p inhibitor significantly suppressed SGC-7901 cell tumorigenesis in vivo. In addition, IHC revealed that CMTM3 expression was markedly increased in tumors infected with miR-135b-5p inhibitor lentivirus. On the whole, the findings of the present study suggest that the overexpression of miR-135b-5p inhibits
\end{abstract}

Correspondence to: Professor Leping Li, Department of General Surgery, Shandong Provincial Hospital Affiliated to Shandong University, 324 Jingwu Road, Ji'nan, Shandong, P.R. China

E-mail: lileping@medmail.com.cn

*Contributed equally

Key words: CKLF-like MARVEL transmembrane domain containing 3, microRNA-135b-5p, gastric cancer, proliferation, invasion
CMTM3 expression, and promotes gastric cancer progression and metastasis. Our findings provide a novel therapeutic target for gastric cancer.

\section{Introduction}

Gastric cancer is the 4th most common type of cancer and is ranked 2nd among the causes of cancer-related mortality worldwide (1-3). Despite significant progress in surgical and chemotherapeutic treatment regimens, the prognosis for patients with gastric cancer remains poor (4). Therefore, there is an urgent need for a better understanding of the molecular mechanisms that underly gastric cancer carcinogenesis, including oncogenes, tumor progression and development, in the hope of discovering novel prognostic markers and therapeutic targets.

The chemokine-like factor (CKLF)-like MARVEL transmembrane domain-containing family (CMTM) is a novel family of proteins that links classical chemokines and the transmembrane- 4 superfamily (5). In humans, CMTM comprises 9 genes, which are CKLF and CMTM1-8 (6). The CMTM family members play diverse role in multiple types of cancer. Among these members, CMTM3, 5, 7 and 8 exhibit tumor suppressive properties in cancers, such as clear cell renal cell carcinoma, oral squamous cell carcinoma, non-small cell lung cancer and hepatocellular carcinoma (7-10). In gastric cancer, the decreased expression of CMTM3 has been observed, and the downregulation of CMTM3 promotes the metastasis of gastric cancer cells. Mechanistic analyses have demonstrated that the knockdown of CMTM3 promotes cancer cell migration and invasion via the epithelial-mesenchymal transition (EMT) process caused by the signal transducer and activator of transcription (STAT)3/Twist1/EMT signaling pathway (11). However, the contribution of CMTM3 to gastric cancer cell proliferation has seldom been discussed, at least to the best of our knowledge. Moreover, although some studies have reported that the promoter hypermethylation inhibits CMTM3 expression in gastric cancer $(12,13)$, the regulatory mechanisms of CMTM3 in gastric cancer require further comprehensive interpretation.

Over the past decades, the contribution of microRNAs (miRNAs or miRs) to oncogenesis and cancer progression 
has been widely discussed. miRNAs are small, endogenous, non-coding RNAs, approximately 20-25 nucleotides in length, that regulate target gene expression at the post-transcriptional level by inhibiting translation or/and cleaving the targeted mRNA by binding to the 3'-untranslated regions (3'-UTRs) of target mRNAs (14). In gastric cancer, investigations on miRNAs and their functions have provided novel targets that may be used for the prediction of the prognosis and for the development of novel therapeutic strategies (15). miR-135b is a well characterized miRNA and has been reported to play a tumor-promoting role in several types of cancer, such as non-small cell lung cancer and breast cancer $(16,17)$. Conversely, other studies have found opposing results, demonstrating that miR-135b inhibits metastasis in prostate cancer and reverses the chemoresistance of non-small cell lung cancer cells by targeting STAT6 and Frizzled-1 (FZD1), respectively $(18,19)$. Moreover, miR-135b-5p has been shown to inhibit lipopolysaccharide (LPS)-induced tumor necrosis factor (TNF)- $\alpha$ production by silencing the AMP-activated protein kinase (AMPK) phosphatase, Ppmle (20). These above-mentioned studies have illustrated the existence of heterogeneity in the function of miR-135b due to its multiple targets in various types of tumors. However, its role in gastric cancer and the relative targets has seldom been discussed previously, at least to the best of our knowledge.

In this study, we first identified that CMTM3 was significantly decreased in gastric cancer tissues, whereas miR-135b-5p was markedly upregulated. We then further investigated the function of CMTM3 and miR-135b-5p in the progression of gastric cancer. Furthermore, we demonstrate that miR-135b-5p promotes gastric cancer progression by targeting CMTM3.

\section{Materials and methods}

Specimens. Gastric cancer tissues and normal adjacent tissues were collected from patients who underwent curative resection at the Department of Surgery, the Second Affiliated Hospital of Wenzhou Medical University, Wenzhou, China between 2013 and 2015. All samples were collected after obtaining written informed consent. The tissues were immediately transported into liquid nitrogen in operating theatres and then stored at $-80^{\circ} \mathrm{C}$. The study protocol was approved by the Ethics Committee of the Second Affiliated Hospital of Wenzhou Medical Univesity, and was carried out according to the Declaration of Helsinki.

Cells and cell culture. The human normal gastric epithelial cell line, GES-1, and the gastric cancer cell lines, SGC-7901, HGC-27, BGC-823 and MKN45, were purchased from the Cell Bank of the Chinese Academy of Sciences. The 293T cells were a kind gift from Wenzhou Medical University. The cells were maintained in DMEM (GES-1, SGC-7901 and 293T), RPMI-1640 medium (BGC-823 and MKN45), or MEM (HGC-27) supplemented with $10 \%$ fetal bovine serum (all from HyClone, Salt Lake City, UT, USA), 100 units/ml penicillin and 100 units/ml streptomycin (Sigma, St. Louis, MO, USA). All these cells were cultured in a humidified environment containing $5 \% \mathrm{CO}_{2}$ and maintained at a constant temperature of $37^{\circ} \mathrm{C}$.
Reverse transcription-quantitative PCR (RT-qPCR). Total RNA for CMTM3 and $\beta$-actin was extracted from the clinical samples using TRIzol reagent (Invitrogen, Carlsbad, CA, USA). The concentration of the RNA was measured using a NanoDrop spectrophotometer at 260/280 nm (Thermo Fisher Scientific, Waltham, MA, USA) and the RNA quality was determined via electrophoresis. First-strand cDNA was synthesized PrimeScript RT Reagent kit (Takara, Mountain View, CA, USA) according to the manufacturer's instructions. Briefly, $4 \mu \mathrm{l}$ of isolated RNA ( $30 \mu \mathrm{g}$ ) was first mixed with $1 \mu \mathrm{l}$ of random hexamer primer and $7 \mu \mathrm{l}$ of RNAse-free $\mathrm{H}_{2} \mathrm{O}$ and then incubated at $65^{\circ} \mathrm{C}$ for $5 \mathrm{~min}$. Subsequently, the microtubes were cooled on ice followed by the addition of $4 \mu \mathrm{l}$ of reaction buffer, $1 \mu \mathrm{l}$ of RNase inhibitor, $2 \mu \mathrm{l}$ of dNTP mix, and $1 \mu \mathrm{l}$ of reverse transcriptase to each sample. The samples were immediately incubated at $25^{\circ} \mathrm{C}$ for $5 \mathrm{~min}$ and then at $42^{\circ} \mathrm{C}$ for $60 \mathrm{~min}$. Finally, the reaction was terminated by heating the samples at $70^{\circ} \mathrm{C}$ for $5 \mathrm{~min}$. The reverse transcription reaction was performed with the final volume of $20 \mu \mathrm{l}$ per tube. Quantitative PCR was performed using SYBR-Green Ex Taq ${ }^{\mathrm{TM}}$ master mix (Takara). The quantitative analysis was carried out using iQ 5 Real-Time PCR System (Bio-Rad, Hercules, CA, USA). The real-time PCR conditions were as follows: $50^{\circ} \mathrm{C}$ for $2 \mathrm{~min}$, $95^{\circ} \mathrm{C}$ for $10 \mathrm{~min}$, then 40 cycles at $95^{\circ} \mathrm{C}$ for $15 \mathrm{sec}$, and $60^{\circ} \mathrm{C}$ for $1 \mathrm{~min}$. The primers used for $\beta$-actin and CMTM3 were as follows: $\beta$-actin forward, 5'-GGCACTCTTCCAGCCTTCC-3'; and reverse, 5'-GAGCCGCCGATCCACAC-3'; and CMTM3 forward, 5'-TCTTGCGTGTGAATCTCTTACC-3'; and reverse, 5'-CAGGATCCACATTGGTGTTACC-3'. Total RNA for miR-135b-5p and U6 was extracted from the clinical samples and cells using the miRNeasy mini kit (Qiagen, Hilden, Germany). The RT-qPCR reactions of miR-135b-5p and U6 were performed according to the manufacturer's instructions of the All-in-One ${ }^{\mathrm{TM}}$ miRNA qRT-PCR Detection kit (GeneCopoeia, Rockville, MD, USA). iQ-5 (Bio-Rad) was used to monitor all these RT-qPCR reactions. RNA expression was relative quantified using $2-\Delta \Delta C t$ method.

Western blot analysis. Total protein was extracted using RIPA protein lysis buffer (Beyotime, Shanghai, China) with $1 \%$ protease inhibitor cocktail and $1 \mathrm{mM}$ phenylmethylsulfonyl fluoride (PMSF). Cell fractions were prepared using a Nuclear and Cytoplasmic Protein Extraction kit (Beyotime) according to the manufacturer's instructions. Generally, $50 \mu \mathrm{g}$ of protein were used for western blotting. Samples were separated by SDS-PAGE and transferred onto PVDF membranes (Thermo Fisher Scientific, Waltham, MA, USA). After blocking in 5\% skim milk, the PVDF membranes were incubated with primary antibodies in blocking buffer overnight at $4^{\circ} \mathrm{C}$ and then with HRP-conjugated secondary antibody for $2 \mathrm{~h}$. The primary antibodies used were as follows: anti- $\beta$-tubulin (1:5,000 dilution, sc-23949; Santa Cruz Biotechnology, Santa Cruz, CA, USA), anti-CMTM3 (1:1,000 dilution, ab198016; Abcam, Cambridge, UK), anti-caspase 3 [1:1,000 dilution, 9662; Cell Signaling Technology (CST), Danvers, MA, USA], anti-poly(ADP-ribose) polymerase (PARP; 1:1,000 dilution, 9532; CST), anti-caspase 9 (1:1,000 dilution, ab32539; Abcam), anti-cyclin B1 (1:1,000 dilution, ab32053; Abcam), anti-p21 (1:1,000 dilution, ab109520; Abcam). The membranes were then incubated with HRP-conjugated goat anti-mouse 
IgG (sc-2005; Santa Cruz Biotechnology) or HRP-conjugated goat anti-rabbit IgG (sc-2004; Santa Cruz Biotechnology). Reactive bands were visualized with ECL reagent (Pierce, Rockford, IL, USA) and analyzed. Protein expression was quantified using ImageJ software (National Institutes of Health, Bethesda, MD, USA).

Immunohistochemistry (IHC). The frozen tumor tissues and adjacent tissues thawed in $4^{\circ} \mathrm{C}$ prior to fixation, and washed with PBS twice. The tissues were then fixed in $3.7 \%$ formalin and embedded in paraffin, and were then cut into $4-\mu \mathrm{m}$-thick serial sections. The paraffin-embedded tissue sections were dewaxed, rehydrated and placed in $10 \mathrm{mmol} / 1$ citrate buffer ( $\mathrm{pH}$ 6.0), and heated twice in a microwave oven for $5 \mathrm{~min}$ each. The sections were incubated with $3 \% \mathrm{H}_{2} \mathrm{O}_{2}$ for $10 \mathrm{~min}$, washed with PBS, blocked with $10 \%$ normal goat serum for $30 \mathrm{~min}$, and then incubated with $4 \mathrm{mg} / \mathrm{l}$ purified anti-CMTM3 (ab198016; Abcam) or normal rabbit IgG (ab172730; Abcam) as a control at $4^{\circ} \mathrm{C}$ overnight. After washing, the sections were stained with the catalyzed signed amplification system kit (Agilent Technologies, Santa Clara, CA, USA) and visualized with a Nikon E800 microscope (Nikon, Tokyo, Japan) and images were acquired.

Oligonucleotide transfection and Luciferase reporter assay. miR-135b-5p mimics and scramble control mimics were purchased from GeneCopoeia Inc. The target gene of miR-135b-5p, was firstly predicted by TargetScan (http:// www.targetscan.org/mamm_31/), miRDB (http://www. mirdb.org/) and miRanda (http://www.microrna.org/), respectively. CMTM3 was then filtered out from the intersection of above 3 prediction results. Wild-type CMTM3 3'UTR (CMTM3-3'UTR-wt) and miR-135B-5p target site deletion mutation CMTM3 3'UTR (CMTM3-3'UTR-mu) were constructed into the psiCHECK2 plasmid (Promega, Madison, WI, USA). The 293 T cells ( $10^{5}$ cells) were seeded in 24-well plates prior to transfection. According to the instructions of the manufacturer, mimics and CMTM3 3'UTR reporter plasmids (psiCHECK-CMTM3-3'UTR-wt or psiCHECK-CMTM3-3'UTR-mu) were co-transfected using Lipofectamine ${ }^{\circledR}$ RNAiMAX (Thermo Fisher Scientific, MA, USA) with a final concentration of $50 \mathrm{nM}$ (mimics) or $200 \mathrm{ng}$ (PrLZ 3'UTR reporter plasmid). After $48 \mathrm{~h}$, the cells were collected and Luciferase activity was detected using the Dual-Glo luciferase assay system (Promega) according to the manufacturer's instructions.

Lentiviral infection. miR-135b-5p inhibitor lentivirus and CMTM3 overexpression lentivirus were purchased from Shanghai R\&S Biotechnology Co., Ltd. The SGC-7901 cells were planted into $10 \mathrm{~cm}$ dishes $\left(10^{6}\right.$ cells/dish) $24 \mathrm{~h}$ prior to infection. Lentiviral infection was performed at a multiplicity of infection (MOI) of 50. The infection efficiency was determined by counting the number of GFP-positive cells which should be guaranteed to be $>90 \%$.

Cell proliferation assay. The cells were seeded in 96-well plates in triplicate at densities of 3,000/well. Cell viability was evaluated at the desired time points using CCK8 kits (Dojindo Molecular Technologies, Kumamoto, Japan) according to the manufacturer's instructions. Light absorbance of the solution was measured at $450 \mathrm{~nm}$ using a microplate reader (PR4100; Bio-Rad).

Cell invasion assay. Transwell chambers coated with Matrigel (BD Biosciences, San Jose, CA, USA) were used for the analysis of cell invasion. A total of $3 \times 10^{5}$ SGC-7901 cells in $100 \mu \mathrm{l}$ serum-free DMEM were seeded on the upper chambers and DMEM with 10\% FBS was added to the lower chambers. After $24 \mathrm{~h}$ of incubation, the invaded cells in the lower side of the membranes were fixed with methanol and stained with crystal violet (Beyotime). Images were acquired using an inverted microscope. Invaded cells were counted from three different fields. The experiment was repeated three times.

Apoptosis assay. For the analysis of cell apoptosis, the transfected cells were harvested, washed and resuspended in $1 \mathrm{ml}$ of binding buffer. The cells were then stained with $5 \mu \mathrm{l}$ of Annexin V-APC (BD Biosciences) and $10 \mu \mathrm{l}$ of propidium iodide (Sigma-Aldrich) in the dark for $15 \mathrm{~min}$ at room temperature and analyzed using a flow cytometer (BD Biosciences) equipped with CellQuest software.

Cell cycle analysis. Propidium iodide (PI) staining with flow cytometry was used to assess cell cycle distribution. Briefly, the lentivirus-infected SGC-7901 cells were released by trypsinization, collected, washed with cold PBS, and then fixed in $70 \%$ ethanol at $4^{\circ} \mathrm{C}$ overnight. The fixed cells were then suspended in $250 \mu \mathrm{l}$ of RNase A buffer $(100 \mathrm{ng} / \mathrm{ml})$, and labeled with a $2 \mathrm{X}$ solution of PI $(100 \mathrm{ng} / \mathrm{ml})$ for $30 \mathrm{~min}$ at $4^{\circ} \mathrm{C}$. Finally, the stained cells were analyzed using a flow cytometer (BD Biosciences) equipped with CellQuest software.

Xenograft mouse model. A total of 30 female BALB/c nude mice (6 weeks old, weighing 18-22 g) were purchased from Nanjing Biomedical Research Institute of Nanjing University (Nanjing, China). These BALB/c nude mice were injected with the SGC-7901 cells subcutaneously (5x106 cells/mouse) into the right posterior shoulder area. For the lentiviral infection groups, $10 \mu 1$ lentivirus were respectively injected into the tumors every 3 days when the tumor volume up had reached up to $50 \mathrm{~mm}^{3}$. The tumor sizes were measured every 3 days using micrometer calipers, and tumor volumes were calculated as follows: Tumor volume $=\mathrm{d}^{2} \mathrm{xD} / 2$, where $\mathrm{d}$ and $\mathrm{D}$ represented shortest and the longest diameters, respectively. At 22 days after the first injection of the lentivirus, the mice were euthanized and tissue was collected. The tissues were immediately transported into liquid nitrogen and then stored at $-80^{\circ} \mathrm{C}$ for subsequent pathology and gene expression detection. All animal experiment protocols were approved by the Institutional Animal Care and Use Committee of Wenzhou Medical University.

Statistical analysis. The results are presented as the means \pm standard deviations (SD) of 3 independent experiments. Significant differences in mean values were evaluated by an unpaired t-test. One-way ANOVA with Tukey's post hoc test was used to compare continuous variables among 2 or more groups. Tests of association were conducted using Pearson's 
A

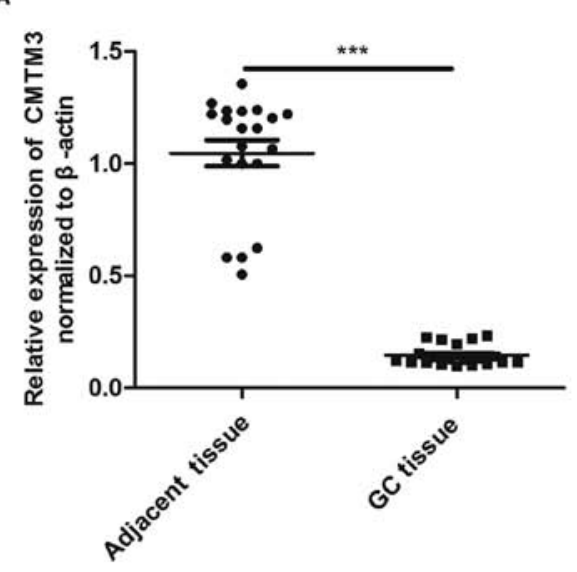

C

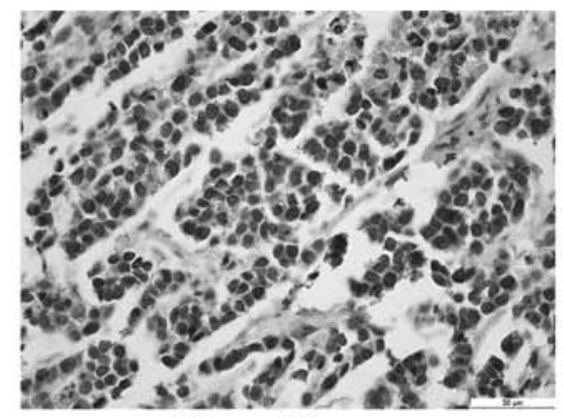

Adjacent tissue

D

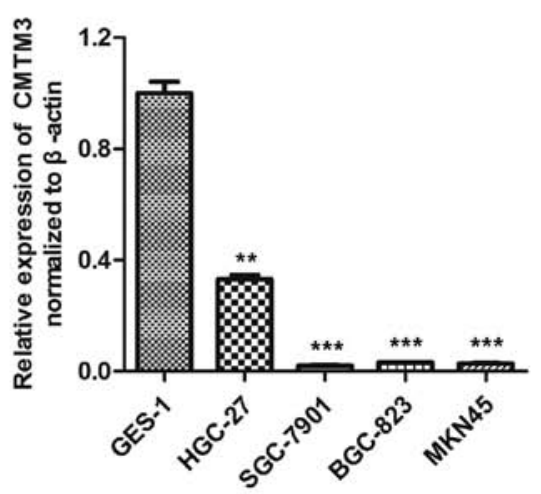

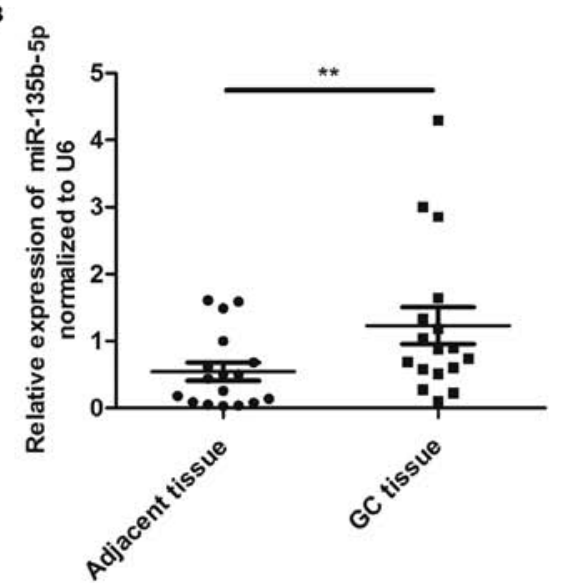

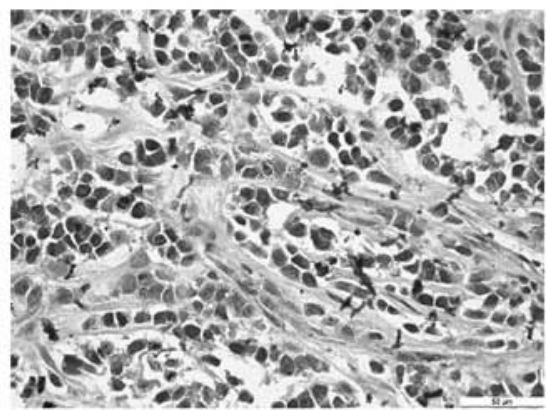

GC tissue

E

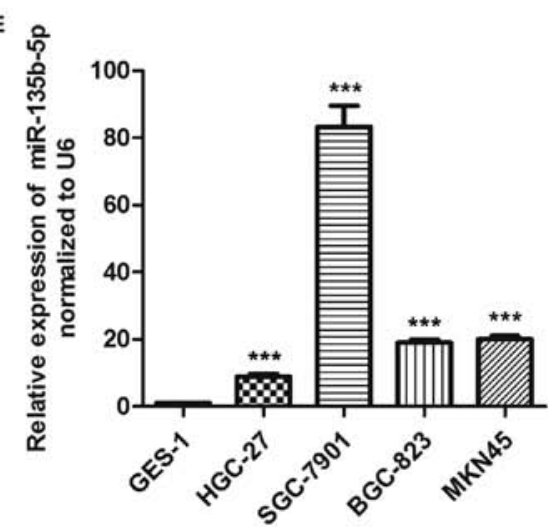

Figure 1. Expression patterns of CMTM3 and miR-135b-5p in gastric cancer tissues and cell lines. (A) Expression of CMTM3 was detected in gastric cancer (GC) tissues and adjacent tissues by RT-qPCR. CMTM3 was significantly downregulated in gastric cancer tissues. ${ }^{* * *} \mathrm{P}<0.001$, shown by paired two-tailed Student's t-test. (B) The miR-135b-5p expression level was markedly higher in gastric cancer tissues compared with adjacent tissues. ${ }^{* *} \mathrm{P}<0.01$, shown by unpaired two-tailed Student's t-test. (C) IHC analysis of CMTM3 in gastric cancer tissues and adjacent tissues. CMTM3 was significantly downregulated in gastric cancer tissues. (D) The expression of CMTM3 was detected in different cell lines by RT-qPCR. CMTM3 expression was decreased in gastric cancer cell lines compared with the GES-1 normal cells. ${ }^{* *} \mathrm{P}<0.01$ and ${ }^{* * *} \mathrm{P}<0.001$, shown by unpaired two-tailed Student's t-test. (E) miR-135b-5p expression level was markedly higher in gastric cancer cells compared with GES-1 cells. ${ }^{* * *} \mathrm{P}<0.001$, shown by unpaired two-tailed Student's t-test.

$\chi^{2}$ test. A value of $\mathrm{P}<0.05$ was considered to indicate a statistically significant difference.

\section{Results}

Expression of CMTM3 and miR-135b-5p in gastric cancer tissues and cells. RT-qPCR and IHC were used to examine the expression pattern of CMTM3 and miR-135b-5p in gastric cancer. The results of RT-qPCR revealed that CMTM3 exhibited a lower expression pattern in gastric cancer tissues compared with the adjacent tissues, while the expression of miR-135b-5p was significantly higher in gastric cancer tissues compared with the adjacent tissues (Fig. 1A and B). Moreover, IHC yielded similar results, also showing that CMTM3 was markedly downregulated in gastric cancer tissues (Fig. 1C). We then performed RT-qPCR to verify the differences in the expression of CMTM3 and miR-135b-5p between the normal gastric epithelial cell line, GES-1, and gastric cancer cell lines (HGC-27, SGC-7901, BGC823 and MKN45). Our results indicated that CMTM3 expression was higher in the GES-1 cells than that in the HGC-27, SGC-7901, BGC823 and MKN45 cells (Fig. 1D). By contrast, the miR-135b-5p expression level was significantly lower in the GES-1 cells compared with the HGC-27, SGC-7901, BGC823 and MKN45 cells (Fig. 1E). 


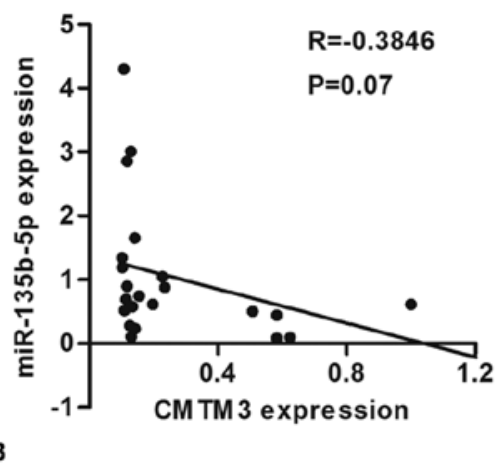

hsa-miR-135b: AGUGUAUCCUUAUUUUUCGGUAU... CMTM3 3'UTR: UCUUACCCUGAAAAAAAGCCAUA' I I I I CMTM3 3'UTR ${ }^{\text {"wt' }}$ : UCUUACCCUGAAAAA-............... AUG

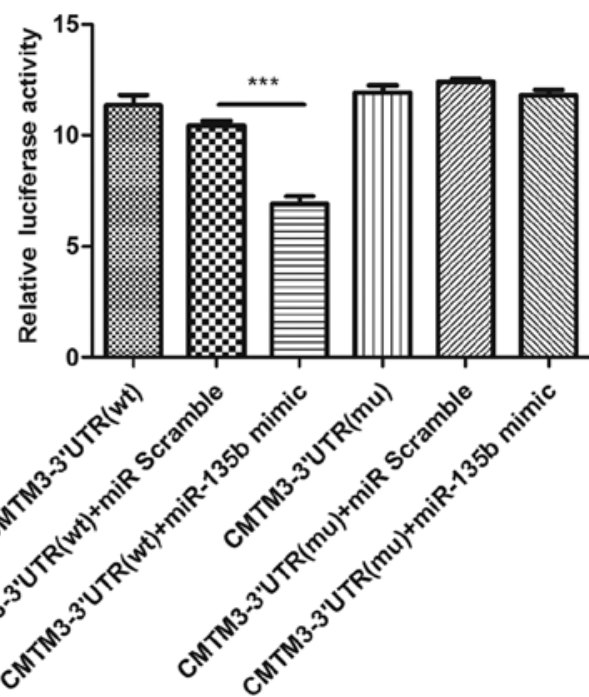

Figure 2. miR-135b-5p targets CMTM3 expression. (A) Correlation analysis of miR-135b-5p and CMTM3 expression in gastric cancer specimens; P=0.07. (B) Target site of miR-135b-5p in CMTM3 3'-UTR predicted combinationaly by TargetScan, miRDB and miRanda. (C) Luciferase activity decreased following transfection with miR-135b-5p mimic in PrLZ-3'-UTR-wt-transfected 293T cells. However, miR-135b-5p mimic was not found in the PrLZ-3'-UTRmu-transfected $293 \mathrm{~T}$ cells. ${ }^{* * *} \mathrm{P}<0.001$, shown by unpaired two-tailed Student's t-test.

miR-135b-5p targets CMTM3 expression. To verify the regulatory association between miR-135b-5p and CMTM3, we first analyzed their expression correlation in gastric cancer tissues. Pearson's correlation analysis revealed that CMTM3 expression negatively correlated with miR-135b-5p expression (Fig. 2A). To identify the potential target site of miR449a, we used a combination of three algorithms, TargetScan, miRDB and miRanda. The target site of miR-135b-5p in CMTM3 3'-UTR predicted by using TargetScan, miRDB and miRanda is shown in Fig. 2B. We further confirmed the target association between miR-135b-5p and CMTM3 by Luciferase assay (Fig. 2C). Our results indicated that CMTM3 was specifically targeted by miR-135b-5p.

CMTM3 inhibits gastric cancer progression. To verify the effect of CMTM3 on gastric cancer cells in vitro, we needed to select a representative cell line. As shown in Fig. 1D and E, CMTM3 expression decreased most significantly in the SGC-7901 cells compared with the GES-1 cells and other gastric cancer cell lines, and miR-135b-5p expression increased most significantly in the SGC-7901 cells. Thus, the SGC-7901 cells were selected for use in the subsequent experiments. CMTM3 overexpression lentivirus was used to introduce the exogenous expression of CMTM3 in the SGC-7901 cells. The effect of CMTM3 overexpression was validated by western blot analysis (Fig. 3A and B). We then performed cell proliferation assay using the CCK-8 proliferation kit. Our results suggested that CMTM3 inhibited SGC-7901 cell proliferation (Fig. 3C). In addition, flow cytometry was used to assess the function of CMTM3 in SGC-7901 cell apoptosis and cell cycle progression, and the results indicated that CMTM3 overexpression significantly promoted SGC-7901 cell apoptosis (Fig. 3D and E). Furthermore, CMTM3 overexpression markedly suppressed SGC-7901 cell cycle progression (Fig. 3F and G). Transwell invasion assays also indicated that CMTM3 overexpression inhibited the invasiveness of the SGC-7901 cells (Fig. 3H and I).
miR-135b-5p inhibitor upregulates CMTM3 and suppresses gastric cancer progression. In order to investigate the function of miR-135b-5p in gastric cancer progression, miR-135b-5p inhibitor lentivirus was used to neutralize miR-135b-5p in the SGC-7901 cells. We found that miR-135b-5p expression was decreased in the SGC-7901 cells following infection with miR-135b-5p inhibitor lentivirus (Fig. 4A), while CMTM3 was significantly upregulated (Fig. 4B and C). Proliferation assay revealed that infection with miR-135b-5p inhibitor inhibited SGC-7901 cell proliferation (Fig. 4D). Flow cytometry was also used to assess the effects of miR-135b-5p inhibitor on SGC-7901 cell apoptosis and cell cycle progression. The results indicated that infection with miR-135b-5p inhibitor significantly promoted SGC-7901 cell apoptosis (Fig. 4E and F). Moreover, infection with miR-135b-5p inhibitor markedly suppressed SGC-7901 cell cycle progression (Fig. 4G and H). Transwell invasion assays also indicated that infection with miR-135b-5p inhibitor suppressed the invasiveness of the SGC-7901 cells (Fig. 4I and J). The above-mentioned results indicated that miR-135b-5p promoted gastric cancer progression by downregulating CMTM3 expression.

The miR-135b-5p/CMTM3 axis regulates indicators of proliferation and apoptosis in SGC-7901 cells. In order to confirm the effects of the miR-135b-5p/CMTM3 axis on cell proliferation, apoptosis and invasion, we further examined the indicators of proliferation, apoptosis and invasion by western blot analysis. The results revealed that CMTM3 overexpression increased the level of cleaved caspase 3, caspase 9 and cleaved PARP in the SGC-7901 cells, which indicated that CMTM3 promoted gastric cancer cell apoptosis (Fig. 5B). The expression of p21, which has been reported to be an indicator of cell cycle arrest (21), was significantly increased after CMTM3 was overexpressed in the SGC-7901 cells. However, the expression of cyclin B1, an important regulatory protein involved in mitosis (22), was markedly decreased in the SGC-7901 cells following infection with 
A

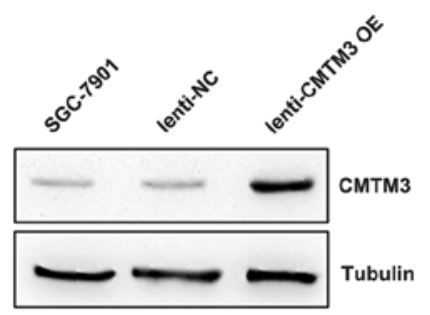

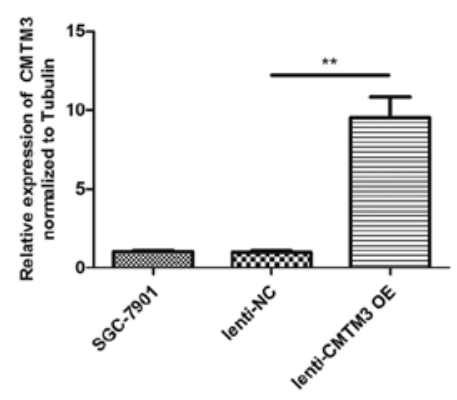

C

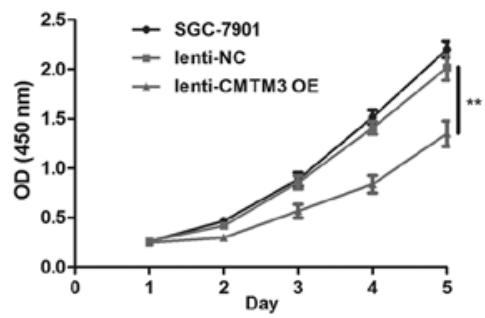

E

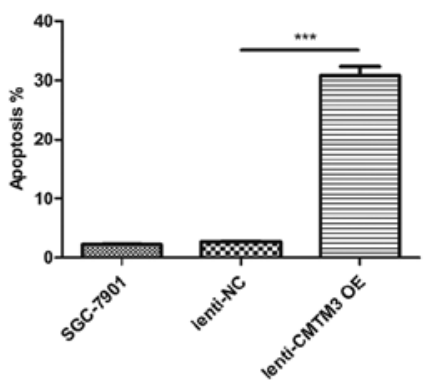

G
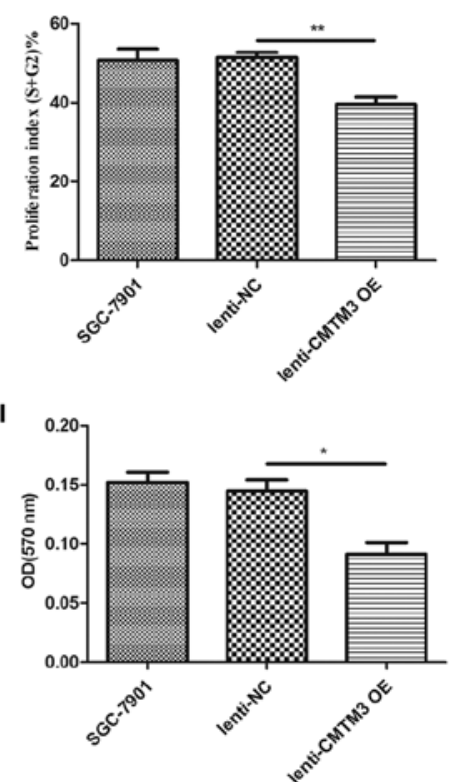

Figure 3. CMTM3 inhibits the malignant phenotype and promotes the apoptosis of SGC-7901 cells. (A and B) CMTM3 expression was detected by western blot analysis in SGC-7901 cells following infection with CMTM3 overexpression lentivirus or negative control (NC) lentivirus. * $\mathrm{P}<0.01$, shown by unpaired two-tailed Student's t-test. (C) CMTM3 overexpression suppressed SGC-7901 cell proliferation. ${ }^{* *} \mathrm{P}<0.01$, shown by one-way ANOVA. (D and E) CMTM3 overexpression promoted SGC-7901 cell apoptosis. ${ }^{* * *}$ P $<0.001$, shown by unpaired two-tailed Student's t-test. (F and G) CMTM3 suppressed SGC-7901 cell cycle progressoin. ${ }^{* *} \mathrm{P}<0.01$, shown by unpaired two-tailed Student's t-test. (H and I) CMTM3 inhibited SGC-7901 cell invasion. ${ }^{*} \mathrm{P}<0.05$, shown by unpaired two-tailed Student's t-test. Group name SGC-7901 indicates untransfected cells.

CMTM3 overexpression lentivirus (Fig. 5B). Moreover, infection with miR-135b-5p inhibitor, which upregulated CMTM3 expression in the SGC-7901 cells, led to a similar effect on the expression of these markers as observed with CMTM3 overexpressoin (Fig. 5A). These results indicated that miR-135b-5p promoted SGC-7901 cell malignant phenotype via targeting CMTM3.

miR-135b-5p inhibitor suppresses SGC-7901 cell tumorigenesis in vivo. Nude mice with SGC-7901 subcutaneously transplanted tumors were used to verify the function of miR-135b-5p in vivo. miR-135b-5p inhibitor lentivirus was used to neutralize miR-135b-5p in the tumors, and we found that the injection of the miR-135b-5p inhibitor lentivirus markedly suppressed tumor formation in vivo (Fig. 6A-C). We further examined miR-135b-5p and CMTM3 in the transplanted tumors from the nude mice, and the results of RT-qPCR revealed that miR-135b-5p expression was decreased in tumors after the injection of miR-135b-5p inhibitor lentivirus (Fig. 6D). Moreover, IHC revealed that CMTM3 expression was markedly increased in the tumors injected with miR-135b-5p inhibitor lentivirus (Fig. 6E). These results indicated that miR-135b-5p promoted gastric cancer progression in vivo. 


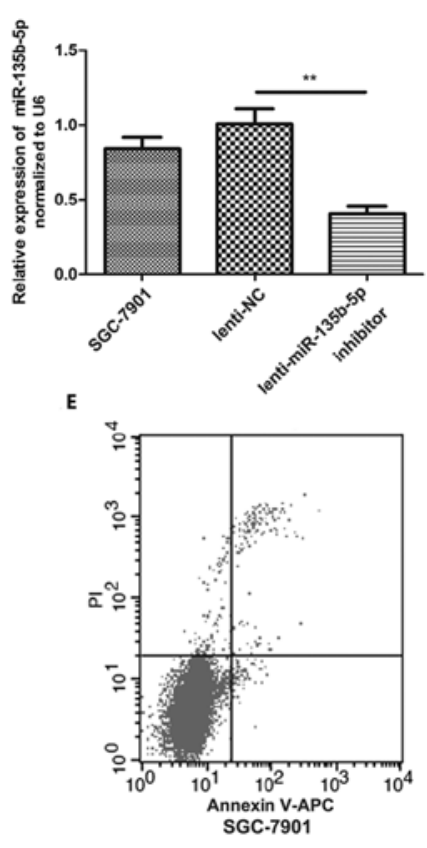

G
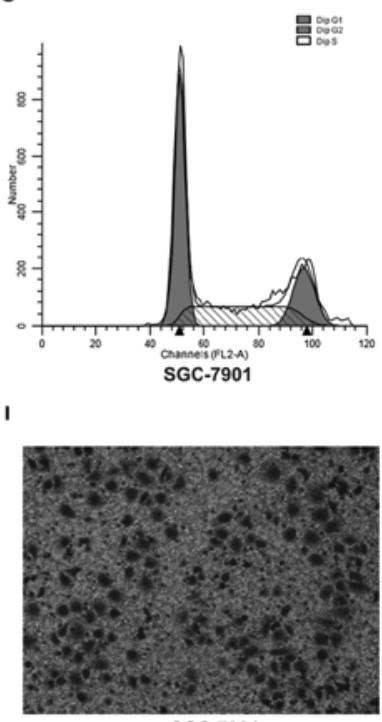
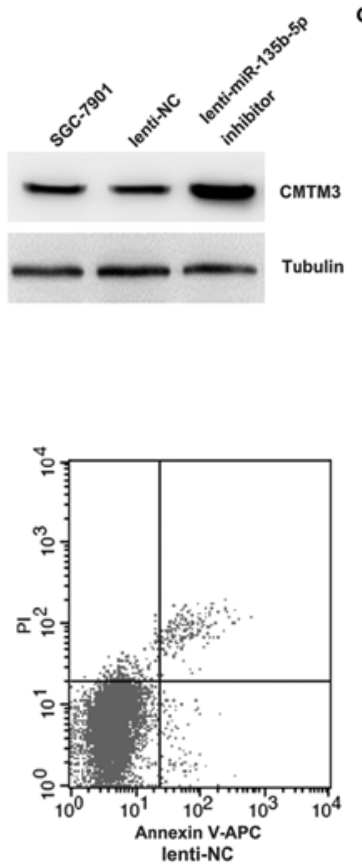

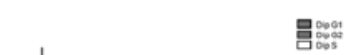
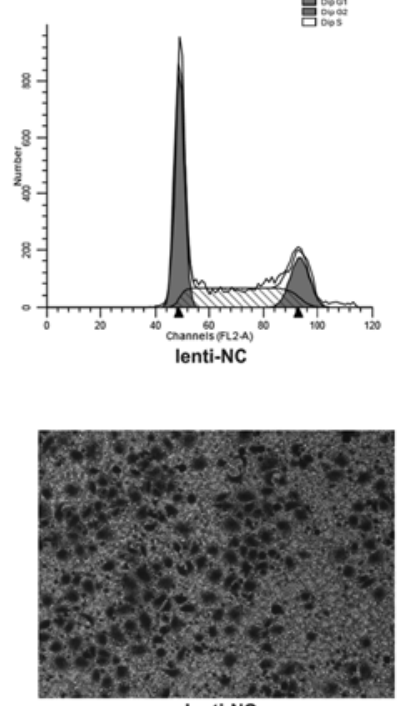
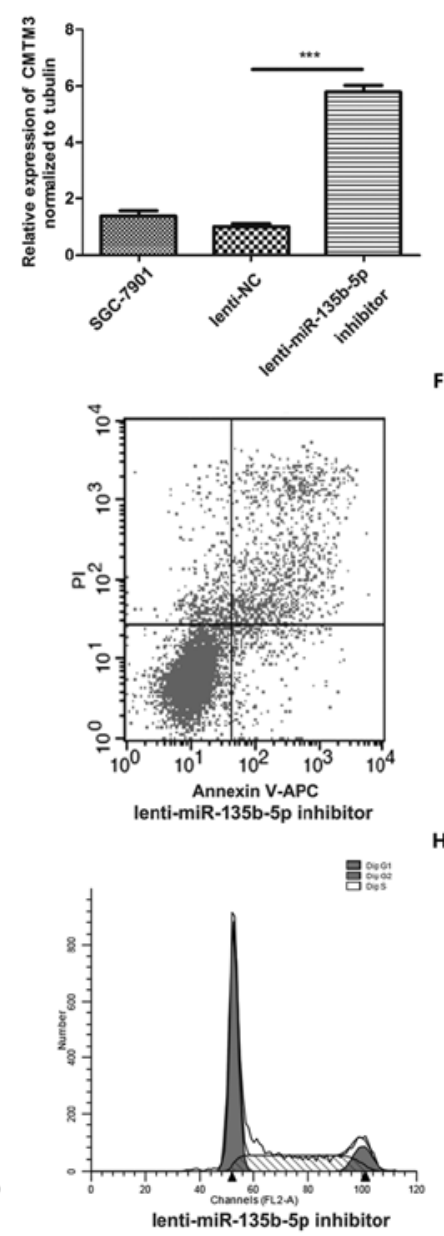

J

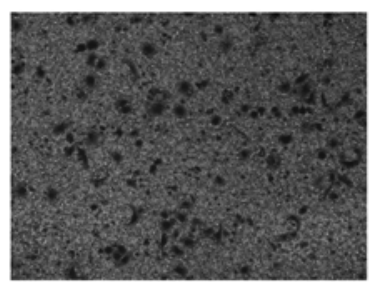

lenti-miR-135b-5p inhibitor
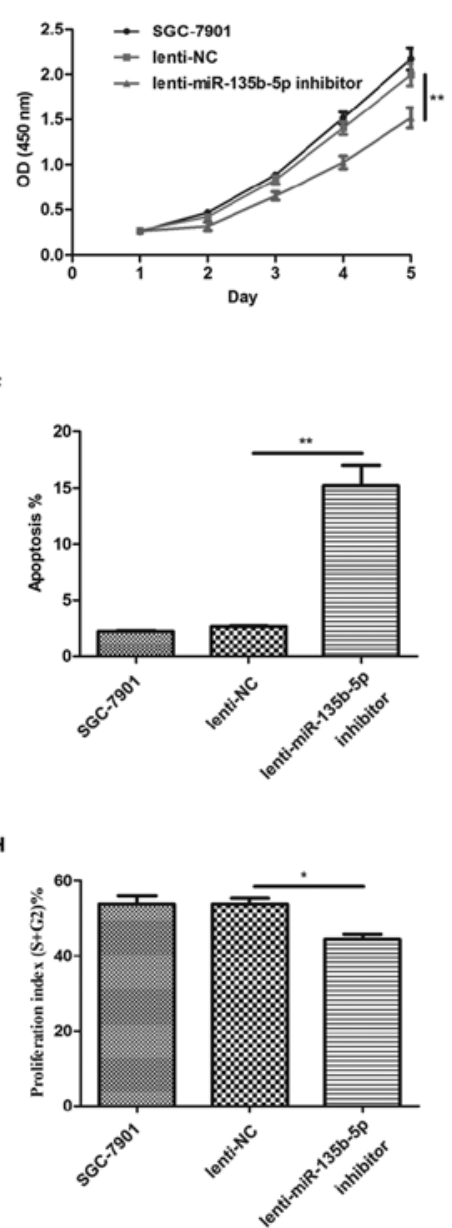

.

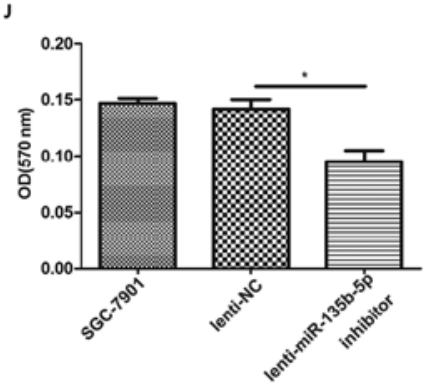

Figure 4. miR-135b-5p inhibitor suppresses malignant phenotypes and promotes the apoptosis of SGC-7901 cells. (A) miR-135b-5p expression was detected by RT-qPCR in SGC-7901 cells following transection with miR-135b-5p inhibitor lentivirus or negative control (NC) lentivirus. ${ }^{* *} \mathrm{P}<0.01$, shown by unpaired two-tailed Student's t-test. (B and C) CMTM3 expresssion was detected by western blot analysis in SGC-7901 cells following infection with miR-135b-5p inhibitor lentivirus or NC lentivirus. ${ }^{* * * *} \mathrm{P}<0.01$, shown by unpaired two-tailed Student's t-test. (D) miR-135b-5p inhibitor suppressed SGC-7901 cell proliferation. ${ }^{* *} \mathrm{P}<0.01$, shown by one-way ANOVA. (E and F) miR-135b-5p inhibitor promoted SGC-7901 cell apoptosis. ${ }^{* *} \mathrm{P}<0.01$, shown by unpaired two-tailed Student's t-test. (G and H) miR-135b-5p inhibitor suppressed SGC-7901 cell cycle progressoin. ${ }^{*} \mathrm{P}<0.05$, shown by unpaired two-tailed Student's t-test. (I and J) miR-135b-5p inhibitor suppressed SGC-7901 cell invasion. " $\mathrm{P}<0.05$, shown by unpaired two-tailed Student's t-test. Group name SGC-7901 indicates untransfected cells.

\section{Discussion}

Despite significant progress being made in surgical techniques and chemotherapeutic regimens for gastric cancer, the death rate remains high. Thus, there is an urgent need for the discovery of novel predictive strategies and therapeutic targets for gastric cancer. In this study, we examined the expression status of CMTM3 and miR-135b-5p in gastric cancer tissues and cell lines, and analyzed the regulatory association between miR-135b-5p and CMTM3. Furthermore, we examined the contribution of miR-135b-5p/CMTM3 axis in gastric cancer progression; our results revealed novel targets for gastric cancer.

CMTMs was shown to play a crucial role as a tumor suppressor in gastric cancer, which is consistent with the findings of previous studies reporting its role in other types of cancer. For example, in prostate cancer, interleukin (IL)-30 treatment has been shown to suppress the expression of CMTM3, leading to the progression of prostate cancer (23). In testicular cancer cells, CMTM3 has been shown to suppress 
A

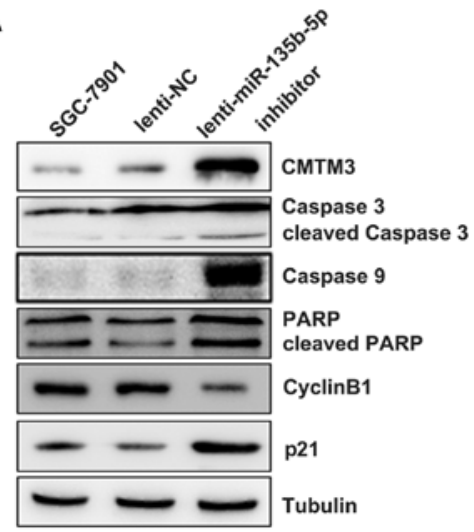

B

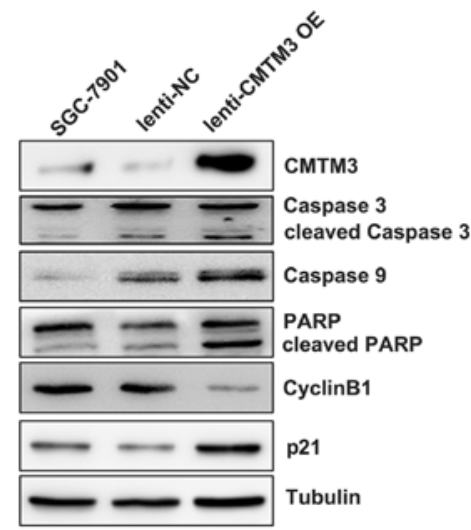

Figure 5. miR-135b-5p/CMTM3 axis regulates indicators of proliferation and apoptosis in SGC-7901 cells. (A) Western blot analysis of apoptosis-related genes (cleaved caspase 3,caspase 9 and cleaved PARP) and cell cycle-related genes (p21 and cyclin B1) in SGC-7901 cells following infection with miR-135b-5p inhibitor lentivirus or negative control (NC) lentivirus. (B) Western blot analysis of apoptosis-related genes (cleaved caspase 3, caspase 9 and cleaved PARP) and cell cycle-related genes (p21 and cyclin B1) in SGC-7901 cells following infection with CMTM3 overexpression lentivirus or NC lentivirus. Group name SGC-7901 indicates untransfected cells.
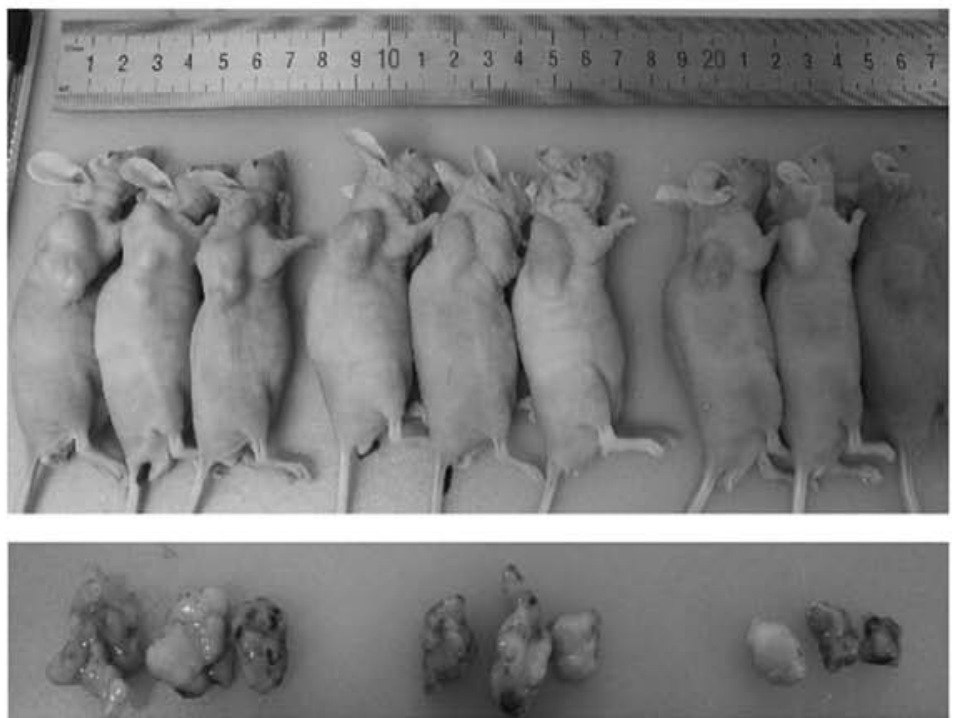

B
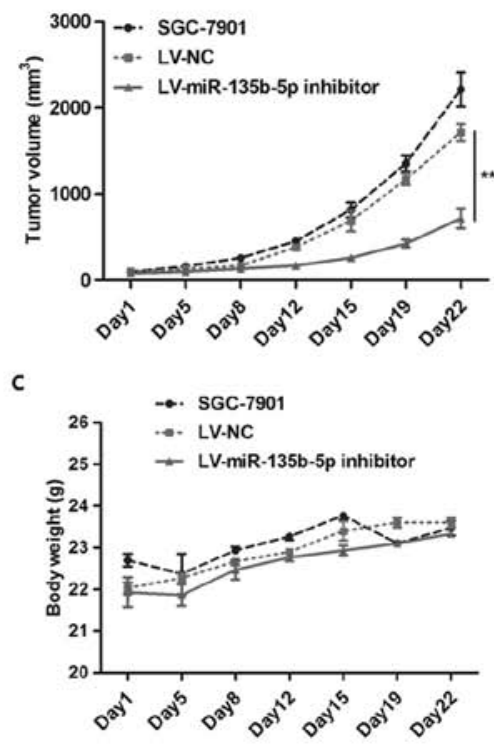

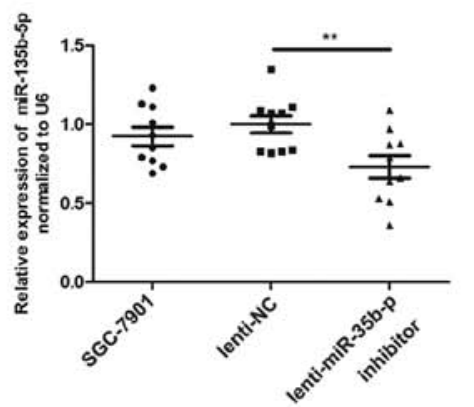

E

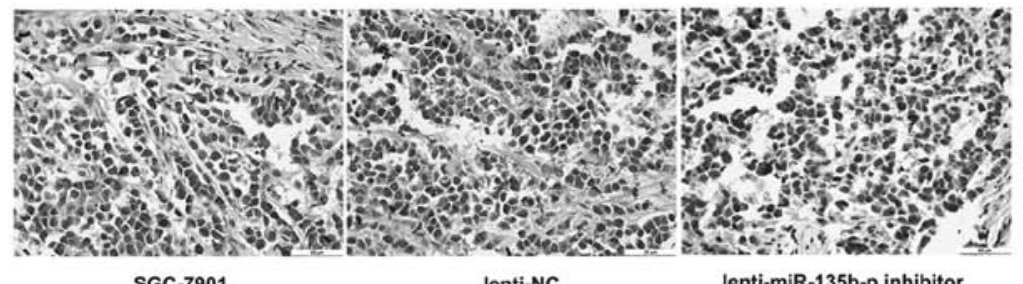

SGC-7901
lenti-NC
lenti-miR-135b-p inhibitor

Figure 6. miR-135b-5p inhibitor suppresses tumor growth and promotes CMTM3 expression in vivo. (A) Representative images of tumors isolated from nude mice. (B) Growth curves of tumors in nude mice. Injection with miR-135b-5p inhibitor lentivirus significantly delayed tumor growth. ${ }^{* * * *} \mathrm{P}<0.001$, shown by one-way ANOVA. (C) Body weight curves of nude mice. (D) RT-qPCR detection of miR-135b-5p in tumors; injection with miR-135b-5p inhibitor lentivirus significantly decreased miR-135b-5p in tumors. ${ }^{* *} \mathrm{P}<0.01$, shown by unpaired two-tailed Student's t-test. (E) IHC analysis of CMTM3. Injection with miR-135b-5p inhibitor lentivirus markedly increased CMTM3 expression in subcutaneous tumors. Group name SGC-7901 indicates untransfected cells.

the proliferation and migration capacities in vitro (24), which is consistent with our observations on gastric cancer cells. A recent study stated the role of CMTM3 in gastric cancer, and stated that the silencing of CMTM3 expression promoted migration and the EMT phenotype of gastric cancer cells.
Mechanistically, CMTM3 regulated the STAT3/Twist1/EMT signaling pathway (13). Although the positive role of CMTM3 in cancer progression has been demonstrated (25), it is well accepted to be a tumor suppressor. Previous studies have suggested that promoter hypermethylation is involved in the 
decreased expression of CMTM3 in various types of cancer, including gastric, colorectal and breast cancer $(11,12,26)$. Currently, miRNA-mediated gene regulation is also considered to be an important aspect of epigenetic regulation in tumorigenesis $(27,28)$. The present study has provided a novel regulatory mechanism of CMTM3 regulation. This promted us to carry out further investigations by the combination of miRNA expression with CMTM3 expression in the diagnosis and treatment of gastric cancer.

miR-135b plays contradictory roles in different types of cancer. For example, in prostate cancer and glioblastoma, miR-135b has been reported to act as a tumor suppressor $(18,29)$. However, in cancers such as breast cancer, hepatocellular carcinoma and colorectal cancer, miR-135b has been reported to be a tumor-promoting factor and to promote cancer cell proliferation and migration $(16,30,31)$. A previous study indicated that the upregulated expression of hsa-miR-135b is found in gastric lesions compared to normal gastric mucosa and intestinal-type gastric adenocarcinoma samples, suggesting the positive role of miR-135b on cancer progression (32). This is consistent with our observations, supporting the positive role of miR-135b in cancer progression. Furthermore, the present study identified a novel target of miR-135b, and broadened the significance of miR-135b in cancers. We verified that miR-135b-5p functioned as an oncogene by targeting CMTM3. Infectoin with miR-135b-5p inhibitor lentivirus induced the upregulation of CMTM3 and increased the apoptotic rate of the SGC-7901 cells, and suppressed SGC-7901 cell proliferation, invasion and cell cycle progression. The results of corresponding western blot analysis for indicator genes, including cleaved caspase 3, caspase 9, cleaved PARP, p21 and cyclin B1 (33-36), further confirmed our findings.

In conclusion, the present study uncovered a novel miR-135b/CMTM3 axis in the progression of gastric cancer, and provided a novel target for the prediction of the prognosis and for the development of novel treatment strategies and therapeutic targets for gastric cancer.

\section{References}

1. Siegel RL, Miller KD and Jemal A: Cancer Statistics, 2017. CA Cancer J Clin 67: 7-30, 2017.

2. Chen W, Zheng R, Baade PD, Zhang S, Zeng H, Bray F, Jemal A, $\mathrm{Yu}$ XQ and He J: Cancer statistics in China, 2015. CA Cancer J Clin 66: 115-132, 2016

3. Liu X, Qiu H, Kong P, Zhou Z and Sun X: Gastric cancer, nutritional status, and outcome. Onco Targets Ther 10: 2107-2114, 2017.

4. Maleki SS and Röcken C: Chromosomal instability in gastric cancer biology. Neoplasia 19: 412-420, 2017.

5. Han W, Lou Y, Tang J, Zhang Y, Chen Y, Li Y, Gu W, Huang J, Gui L, Tang Y, et al: Molecular cloning and characterization of chemokine-like factor 1 (CKLF1), a novel human cytokine with unique structure and potential chemotactic activity. Biochem $\mathrm{J}$ 357: 127-135, 2001.

6. Han W, Ding P, Xu M, Wang L, Rui M, Shi S, Liu Y, Zheng Y, Chen Y, Yang T, et al: Identification of eight genes encoding chemokine-like factor superfamily members 1-8 (CKLFSF1-8) by in silico cloning and experimental validation. Genomics 81: 609-617, 2003

7. Xie J, Yuan Y, Liu Z, Xiao Y, Zhang X, Qin C, Sheng Z, Xu T and Wang X: CMTM3 is frequently reduced in clear cell renal cel carcinoma and exhibits tumor suppressor activities. Clin Trans Oncol 16: 402-409, 2014.

8. Shao L, Guo X, Plate M, Li T, Wang Y, Ma D and Han W: CMTM5-v1 induces apoptosis in cervical carcinoma cells. Biochem Biophys Res Commun 379: 866-871, 2009.
9. Liu B, Su Y, Li T, Yuan W, Mo X, Li H, He Q, Ma D and Han W: CMTM7 knockdown increases tumorigenicity of human non-small cell lung cancer cells and EGFR-AKT signaling by reducing Rab5 activation. Oncotarget 6: 41092-41107, 2015.

10. Zhang W, Mendoza MC, Pei X, Ilter D, Mahoney SJ, Zhang Y, Ma D, Blenis $\mathbf{J}$ and Wang Y: Down-regulation of CMTM8 induces epithelial-to-mesenchymal transition-like changes via c-MET/extracellular signal-regulated kinase (ERK) signaling. J Biol Chem 287: 11850-11858, 2012.

11. Yuan W, Li T, Mo X, Wang X, Liu B, Wang W, Su Y, Xu L and Han W: Knockdown of CMTM3 promotes metastasis of gastric cancer via the STAT3/Twist1/EMT signaling pathway. Oncotarget 7: 29507-29519, 2016.

12. Wang Y, Li J, Cui Y, Li T, Ng KM, Geng H, Li H, Shu XS, Li H, Liu W, et al: CMTM3, located at the critical tumor suppressor locus 16q22.1, is silenced by $\mathrm{CpG}$ methylation in carcinomas and inhibits tumor cell growth through inducing apoptosis. Cancer Res 69: 5194-5201, 2009.

13. Su Y, Lin Y, Zhang L, Liu B, Yuan W, Mo X, Wang X, Li H, Xing $\mathrm{X}$, Cheng $\mathrm{X}$, et al: CMTM3 inhibits cell migration and invasion and correlates with favorable prognosis in gastric cancer. Cancer Sci 105: 26-34, 2014.

14. Catela Ivkovic T, Voss G, Cornella H and Ceder Y: microRNAs as cancer therapeutics: A step closer to clinical application. Cancer Lett 407: 113-122, 2017.

15. Zheng Q, Chen C, Guan H, Kang W and Yu C: Prognostic role of microRNAs in human gastrointestinal cancer: A systematic review and meta-analysis. Oncotarget 8: 46611-46623, 2017.

16. Hua K, Jin J, Zhao J, Song J, Song H, Li D, Maskey N, Zhao B, Wu C, Xu H, et al: miR-135b, upregulated in breast cancer, promotes cell growth and disrupts the cell cycle by regulating LATS2. Int J Oncol 48: 1997-2006, 2016.

17. Xue Y, Ni T, Jiang Y and Li Y: Long noncoding RNA GAS5 inhibits tumorigenesis and enhances radiosensitivity by suppressing miR-135b expression in non-small cell lung cancer. Oncol Res 25: 1305-1316, 2017.

18. Wang N, Tao L, Zhong H, Zhao S, Yu Y, Yu B, Chen X, Gao J and Wang R: miR-135b inhibits tumour metastasis in prostate cancer by targeting STAT6. Oncol Lett 11: 543-550, 2016.

19. Su W, Mo Y, Wu F, Guo K, Li J, Luo Y, Ye H, Guo H, Li D and Yang Z: miR-135b reverses chemoresistance of non-small cell lung cancer cells by downregulation of FZD1. Biomed Pharmacother 84: 123-129, 2016.

20. Li P, Fan JB, Gao Y, Zhang M, Zhang L, Yang N and Zhao X: miR-135b-5pinhibits LPS-induced TNF $\alpha$ production via silencing AMPK phosphatase Ppmle. Oncotarget 7: 77978-77986, 2016.

21. Li Z, Li X, Xu L, Tao Y, Yang C, Chen X, Fang F, Wu Y, DingX, Zhao $\mathrm{H}$, et al: Inhibition of neuroblastoma proliferation by PF-3758309, a small-molecule inhibitor that targets p21-activated kinase 4. Oncol Rep 38: 2705-2716, 2017.

22. Miles DC, van den Bergen JA, Sinclair AH and Western PS: Regulation of the female mouse germ cell cycle during entry into meiosis. Cell Cycle 9: 408-418, 2010.

23. Di Meo S, Airoldi I, Sorrentino C, Zorzoli A, Esposito S and Di Carlo E: Interleukin-30 expression in prostate cancer and its draining lymph nodes correlates with advanced grade and stage. Clin Cancer Res 20: 585-594, 2014.

24. Li Z, Xie J, Wu J, Li W, Nie L, Sun X, Tang A, Li X, Liu R, Mei H, et al: CMTM3 inhibits human testicular cancer cell growth through inducing cell-cycle arrest and apoptosis. PLoS One 9: e88965, 2014.

25. Delic S, Thuy A, Schulze M, Proescholdt MA, Dietrich P, Bosserhoff AK and Riemenschneider MJ: Systematic investigation of CMTM family genes suggests relevance to glioblastoma pathogenesis and CMTM1 and CMTM3 as priority targets. Genes Chromosomes Cancer 54: 433-443, 2015.

26. Li J, Chen C, Bi X, Zhou C, Huang T, Ni C, Yang P, Chen S, Ye M and Duan S: DNA methylation of CMTM3, SSTR2, and MDFI genes in colorectal cancer. Gene 630: 1-7, 2017.

27. Cho CJ, Myung SJ and Chang S: ADAR1 and microRNA; A hidden crosstalk in cancer. Int J Mol Sci 18: E799, 2017.

28. Forrest ME and Khalil AM: Review: Regulation of the cancer epigenome by long non-coding RNAs. Cancer Lett 407: 106-112, 2017.

29. Lulli V, Buccarelli M, Martini M, Signore M, Biffoni M, Giannetti S, Morgante L, Marziali G, Ilari R, Pagliuca A, et al: miR-135b suppresses tumorigenesis in glioblastoma stem-like cells impairing proliferation, migration and self-renewal. Oncotarget 6: 37241-37256, 2015. 
30. Li J, Liang H, Bai M, Ning T, Wang C, Fan Q, Wang Y, Fu Z, Wang N, Liu R, et al: miR-135b promotes receptor II (TGFBR2) in colorectal cancer. PLoS One 10: e0130194, 2015.

31. Li Y, Xu D, Bao C, Zhang Y, Chen D, Zhao F, Ding J, Liang L, Wang Q, Liu L, et al: MicroRNA-135b, a HSF1 target, promotes tumor invasion and metastasis by regulating RECK and EVI5 in hepatocellular carcinoma. Oncotarget 6: 2421-2433, 2015.

32. Vidal AF, Cruz AM, Magalhães L, Pereira AL, Anaissi AK Alves NC, Albuquerque PJ, Burbano RM, Demachki S and Ribeiro-dos-Santos Â: hsa-miR-29c and hsa-miR-135b differential expression as potential biomarker of gastric carcinogenesis. World J Gastroenterol 22: 2060-2070, 2016.

33. Suzuki H, Maruyama R, Yamamoto E and Kai M: Epigenetic alteration and microRNA dysregulation in cancer. Front Genet 4: $258,2013$.
34. Ando T, Yoshida T, Enomoto S, Asada K, Tatematsu M, Ichinose M, Sugiyama T and Ushijima T: DNA methylation of microRNA genes in gastric mucosae of gastric cancer patients: Its possible involvement in the formation of epigenetic field defect. Int J Cancer 124: 2367-2374, 2009.

35. Zhang X, Zhao X, Fiskus W, Lin J, Lwin T, Rao R, Zhang Y, Chan JC, Fu K, Marquez VE, et al: Coordinated silencing of MYC-mediated miR-29 by HDAC3 and EZH2 as a therapeutic target of histone modification in aggressive B-Cell lymphomas. Cancer Cell 22: 506-523, 2012.

36. Yan $\mathrm{H}$, Choi AJ, Lee $\mathrm{BH}$ and Ting $\mathrm{AH}$ : Identification and functional analysis of epigenetically silenced microRNAs in colorectal cancer cells. PLoS One 6: e20628, 2011. 